\title{
Analysis of Call Admission Control with IM Service in Multimedia OFDMA System
}

\author{
Yi Chen \\ Department of Information Science \& \\ Electronic Engineering, \\ Zhejiang University, Hangzhou, China \\ 21131028@zju.edu.cn
}

\author{
Zhifeng Ni \\ Department of Information Science \& \\ Electronic Engineering, \\ Zhejiang University, Hangzhou, China \\ nzf945@zju.edu.cn
}

\author{
Yong Wang \\ Department of Information Science \& \\ Electronic Engineering, \\ Zhejiang University, Hangzhou, China \\ wangy@zju.edu.cn
}

\begin{abstract}
This paper presents an appropriate call admission control (CAC) scheme which admits Instant Message (IM) traffic with conventional traffic for the next-generation OFD MA wireless network. In the proposed scheme, we consider three types of traffic having different quality-of-service (QoS) requirement, namely guaranteed traffic, IM traffic and data traffic. Due to the priority of IM traffic between that of guaranteed traffic and data traffic, we propose a double -threshold guard channel policy and an analytical model with fractional guard channel policy. On the premise of guaranteeing the call-level performance of conventional traffic, the optimized QoS parameters of IM traffic can be obtained by tuning the guard channel dynamically. Simulation results show QoS parameters of traffic and verify the accuracy of the analysis.
\end{abstract}

Keywords-call admission control; OFDMA; IM traffic; quality-of-service

\section{INTRODUCTION}

In recent years, instant messaging (IM) has become increasingly popular. It is estimated that there are several millions of instant messaging users in the world [1] due to its quick response time, its ease of use, and possibility of multitasking. However, with IM service, the rapidly growing multimedia services make a great demand for bandwidth on OFDMA cellular wireless network. Bandwidth is the most critical resource in wireless networks, thus, both efficient call admission control (CAC) and packet scheduling can cooperate to guarantee quality-of-service (QoS) of multimedia services and allocate bandwidth efficiently. For our work, we mainly draw the attention to the research of CAC in the call level.

Up to present, many admission control and resource management schemes for IEEE 802.16e have been proposed in [2-5]. IEEE 802.16e Broadband Wireless Access (BWA) system is developed based on IEEE 802.16d, and it enables a network for fixed and mobile broadband wireless access. The IEEE 802.16e standard defines five types of service classes which are characterized by the different levels of QoS requirements: unsolicited grant service (UGS), real-time polling service (rtPS), extended real-time polling service (ertPS), non-real-time polling service (nrtPS) and best effort service (BE). In [2] HY Tung and KF Tsang proposed a total QoS solution including dynamic call admission control scheme and bandwidth allocation algorithm for IEEE 802.16e mobile WiMAX. In this paper, the authors investigated the relationship between the channel utilization, the dropping and blocking probability versus traffic loads. In [3] Hui-Juan Yao and G.S. Kuo proposed a QoS-adaptive admission control with resource management which is undefined in IEEE 802.16e standard. Based on the different types of scheduling service in IEEE 802.16e standard, the proposed scheme classifies them into different according to handoff-related parameters and uses different bandwidth reservation strategies to guarantee their connection-level QoS. In [4] the authors proposed a dynamic bandwidth reservation admission control scheme for IEEE 802.16e system and an analytical traffic model to evaluate the scheme. They set some overall reserved resource as admission guard bandwidth, which is dynamically adjusted according to the bandwidth request of ongoing real-time variable-bit rate services in addition to guaranteeing the resource requirement of potential handoff services. In [5] Kalikivayi Suresh and Kalpana saha proposed an efficient CAC scheme for IEEE 802.16e Mobile WiMAX that satisfies both bandwidth and delay guarantee to the admitted connections. The proposed CAC scheme provides higher priority than new connections, and UGS connections have the higher priority than other services connections. An analytical model is developed to evaluate the performance of the CAC scheme.

For the next-generation OFDMA wireless network, paper [6] provides a CAC algorithm dedicated to support voice over IP service, in which the VoIP traffic with different priorities will have different admission strategies and therefore different performance. In [7, 8] authors take the users' mobility in one cell into consideration and propose a call admission control reservation algorithm about resource fluctuations.

To the best of authors knowledge, the CAC schemes proposed in above references didn't considerate the IM traffic. Thus, in this paper, we propose an appropriate CAC scheme which admits the IM traffic with the conventional traffic for the OFDMA wireless network. Due to the priority of IM traffic between that of guaranteed traffic and data traffic, we can classify the conventional services defined in IEEE $802.16 \mathrm{e}$ into two services: guaranteed service and data service. According to the priority order, we propose a double-threshold guard channel policy and an analytical 
model with fractional guard channel policy [9].

The remainder of this paper is organized as follows. In section II, we describe the system model. In section III, we present the proposed CAC policy. In section IV, we propose the analytical model. In section $\mathrm{V}$, we give the results of simulations. Finally, conclusions are drawn in VI.

\section{SYSTEM MODEL}

Consider an OFDMA cellular network supporting multiple services. We define $C$ as the number of subchannels, and $R_{b}$ as the average data per subchannel in a cell. We categorize the call connections in the network into tree types: guaranteed service, IM service and data service, represented by $U_{1}, U_{2}$ and $U_{3}$. Let $r_{1}, r_{2}$ and $r_{3}$ respectively denotes the average bandwidth resource (in subchannel) which are requested by each call of classes $U_{1}, U_{2}$ and $U_{3}$. And $n_{i}, i=1,2,3$, respectively presents the number of the call connections of classes $U_{1}, U_{2}$ and $U_{3}$ in a cell. Traffic load of the whole network can be expressed as

$$
R(S)=\sum_{i=1}^{3} n_{i} r_{i}
$$

Assuming that the call generating processes of all the users in a cell are independent, users in the cell generate a connection is a Poisson process with the mean rate $\lambda_{n}$, and the new connection belongs to $U_{1}, U_{2}$ and $U_{3}$ with the probability $p_{1}, p_{2}$ and $p_{3}$,where $p_{1}+p_{2}+p_{3}=1$. Thus the new call with traffic type $U_{i}$ arrives to the cell with the arrival rate of $\lambda_{i, n}=\lambda_{n} \cdot p_{i}, i=1,2,3$. Considering that the movement of each user is modeled by random walk and is stationary process [10], the cell residual time is an exponential distribution with mean $1 / \gamma$, the mean residual time is assumed to be proportional to the cell radius $R$ [11], i.e. $1 / \gamma=\alpha R, \alpha$ is a mobility dependent parameter which must be determined for each mobility setting. The call holding time is exponentially distributed with a mean of $1 / \mu_{i}, i=1,2,3$. According to [11], the handoff call occur with Poisson process, and the rate is donated by $\lambda_{i, h}$ which is given by $\lambda_{i, h}=\lambda_{i, n} \gamma / \mu_{i}, i=1,2,3$, where the second term in right side is the ratio of the mean call holding time to the mean residual time.

Every type of services includes two call requests: new call request and handoff call request. We hope the priority of handoff calls is higher than that of new calls. In conventional scheme, a set of channels called the guard channel are permanently reserved for handoff calls. Thus, handoff calls get more channels than new calls. In this paper, due to having reserved the guard channel to different type of services which is described in Section III, we adopt the fractional guard channel policy to make these two calls connect into a cell. When the traffic load is $R(S)$, the new call can be generated with the probability $\beta_{i, R(S)}$, and $i=1,2,3$ respectively denotes the type of services, and the handoff call can be connected into a cell with the probability 1 .

\section{PROPOSED CAC POLICY}

IM service is a special class of service due to its priority is higher than that of data service and lower than that of guaranteed service, where we can define their priorities as $U_{1}>U_{2}>U_{3}$. In this paper, according to the priority order, we propose a double-threshold guard channel policy. Within the total $C$ subchannels in a cell, we can get the guard channel as $0<G_{2}<G_{1}<C$, where $G_{1}$ and $G_{2}$ denote the number of guard channels. When traffic load of the whole network is $0<R(S)<G_{2}$, new calls of $U_{1}, U_{2}$ and $U_{3}$ can be admitted with the probability $\beta_{i, R(S)}, i=1,2,3$, and handoff calls can be admitted with probability 1 . When traffic load of the whole network is $G_{2} \leq R(S)<G_{1}$, new calls of $U_{1}$ and $U_{2}$ can be admitted with the probability $\beta_{i, R(S)}, i=1,2$, and handoff calls can be admitted with probability 1 . When traffic load of the whole network is $G_{1} \leq R(S)<C$, new calls of $U_{1}$ can be admitted with the probability $\beta_{1, R(S)}$, and handoff calls can be admitted with probability 1 . When traffic load of the whole network is $R(S)=C$, calls of $U_{1}, U_{2}$ and $U_{3}$ cannot be admitted into the network absolutely.

\section{ANALYTICAL MODEL}

\section{A. System State Description}

The performance analysis in our CAC scheme can be done based on the Continuous Time Markov Chain Model. In this model, the state of system can be presented as $\left(n_{1}, n_{2}, n_{3}\right)$. The state space of the Markov Chain is obtained based on the CAC scheme proposed in Section III, thus, the state space $S$ can be expressed as

$$
\begin{aligned}
& S=\left\{\left(n_{1}, n_{2}, n_{3}\right)\right. \\
& \mid\left(n_{1} r_{1}+n_{2} r_{2}+n_{3} r_{3} \leq C\right),\left(n_{2} r_{2}+n_{3} r_{3} \leq G_{1}\right) \\
& \left.,\left(n_{3} r_{3} \leq G_{2}\right)\right\}
\end{aligned}
$$

For the given state $s=\left(n_{1}, n_{2}, n_{3}\right)$, the state transition occurs when a new request for call admission is accepted or an ongoing call terminates. The new request for call admission can be either from handoff call from the neighboring cell or a newly originated call in this cell.

\section{B. State Transition Analysis}

The events that trigger the transition of system state can be classified into four categories: 1) new call arrival; 2) handoff call arrival; 3) handoff call outward; 4) call termination. We describe each category as follows.

\section{1) New Call Arrival}

a. New $U_{1}$ Call Arrivals: When a new $U_{1}$ call arrivals in the cell, it will be accepted if $R(S)+r_{1} \leq C$, and $\left(n_{1}, n_{2}, n_{3}\right) \rightarrow\left(n_{1}+1, n_{2}, n_{3}\right)$, with a rate of $\lambda_{1, n}$. $\beta_{1, R(S)}$. Otherwise, the call is blocked.

b. New $U_{2}$ Call Arrivals: When a new $U_{2}$ call arrivals in the cell, it will be accepted if $R(S)+r_{2} \leq G_{1}$, and $\left(n_{1}, n_{2}, n_{3}\right) \rightarrow\left(n_{1}, n_{2}+1, n_{3}\right)$, with a rate of $\lambda_{2, n}$. $\beta_{2, R(S)}$. Otherwise, the call is blocked. 
c. New $U_{3}$ Call Arrivals: When a new $U_{3}$ call arrivals in the cell, it will be accepted if $R(S)+r_{3} \leq G_{2}$, and $\left(n_{1}, n_{2}, n_{3}\right) \rightarrow\left(n_{1}, n_{2}, n_{3}+1\right)$, with a rate of $\lambda_{3, n}$. $\beta_{3, R(S)}$. Otherwise, the call is blocked.

\section{2) Handoff Call Arrival}

a. Handoff $U_{1}$ Call Arrivals: When a handoff $U_{1}$ call arrivals in the cell, it will be accepted if $R(S)+r_{1} \leq C$, and $\left(n_{1}, n_{2}, n_{3}\right) \rightarrow\left(n_{1}+1, n_{2}, n_{3}\right)$, with a rate of $\lambda_{1, h}=\lambda_{1, n} \gamma / \mu_{1}$. Otherwise, the call is blocked.

b. Handoff $U_{2}$ Call Arrivals: When a handoff $U_{2}$ call arrivals in the cell, it will be accepted if $R(S)+r_{2} \leq G_{1}$, and $\left(n_{1}, n_{2}, n_{3}\right) \rightarrow\left(n_{1}, n_{2}+1, n_{3}\right)$, with a rate of $\lambda_{2, h}=\lambda_{2, n} \gamma / \mu_{2}$. Otherwise, the call is blocked.

c. Handoff $U_{3}$ Call Arrivals: When a handoff $U_{3}$ call arrivals in the cell, it will be accepted if $R(S)+r_{3} \leq G_{2}$, and $\left(n_{1}, n_{2}, n_{3}\right) \rightarrow\left(n_{1}, n_{2}, n_{3}+1\right)$, with a rate of $\lambda_{3, h}=\lambda_{3, n} \gamma / \mu_{3}$. Otherwise, the call is blocked.

\section{3) Handoff Call Outward}

When a user with calls moves to the neighboring cell, bandwidth resource will be released within this cell. If the call belongs to a user in $U_{1},\left(n_{1}, n_{2}, n_{3}\right) \rightarrow\left(n_{1}-1, n_{2}, n_{3}\right)$ with a rate of $n_{1} \gamma$. If the call belongs to a user in $U_{2}$, $\left(n_{1}, n_{2}, n_{3}\right) \rightarrow\left(n_{1}, n_{2}-1, n_{3}\right)$ with a rate of $n_{2} \gamma$. If the call belongs to a user in $U_{3},\left(n_{1}, n_{2}, n_{3}\right) \rightarrow\left(n_{1}, n_{2}, n_{3}-1\right)$ with a rate of $n_{3} \gamma$.

4) Call Termination

When a call belonging to a user in $U_{1}$ terminates, $\left(n_{1}, n_{2}, n_{3}\right) \rightarrow\left(n_{1}-1, n_{2}, n_{3}\right)$, and the call termination occurs with a rate of $n_{1} \mu_{1}$. When a call belonging to a user in $U_{2}$ terminates, $\left(n_{1}, n_{2}, n_{3}\right) \rightarrow\left(n_{1}, n_{2}-1, n_{3}\right)$, and the call termination occurs with a rate of $n_{2} \mu_{2}$. When a call belonging to a user in $U_{3}$ terminates, $\left(n_{1}, n_{2}, n_{3}\right) \rightarrow$ $\left(n_{1}, n_{2}, n_{3}-1\right)$, and the call termination occurs with a rate of $n_{3} \mu_{3}$.

\section{Performance Measures}

By analyzing state transition and using the normalized condition $\sum_{s \in S} \pi(s)=1$, the steady state probability $\{\pi(S)\}$ of all states in state space $S$ can be obtained. From the steady state probability, we can determine QoS parameters of the performance analysis.

\section{1) New Call Blocking Probability}

The new call blocking probability for $U_{1}$ connections $P_{1, n b}$ can be expressed as

$$
P_{1, n b}=\sum_{S \in \psi_{1}} \pi(S)+\sum_{S \in \bar{\psi}_{1}} \pi(S)\left(1-\beta_{1, R(S)}\right)
$$

where $\psi_{1}=\left\{S \mid R(S)+r_{1}>C\right\}$.

The new call blocking probability for $U_{2}$ connections $P_{2, n b}$ can be expressed as

$$
P_{2, n b}=\sum_{S \in \psi_{2}} \pi(S)+\sum_{S \in \bar{\psi}_{2}} \pi(S)\left(1-\beta_{2, R(S)}\right)
$$

where $\psi_{2}=\left\{S \mid R(S)+r_{2}>G_{1}\right\}$.

The new call blocking probability for $U_{3}$ connections
$P_{3, n b}$ can be expressed as

$$
P_{3, n b}=\sum_{S \in \psi_{3}} \pi(S)+\sum_{S \in \bar{\psi}_{3}} \pi(S)\left(1-\beta_{3, R(S)}\right)
$$

where $\psi_{3}=\left\{S \mid R(S)+r_{3}>G_{2}\right\}$.

By using the above probabilities, the overall new blocking probability is given by

$$
P_{n b}=P_{1, n b} \cdot p_{1}+P_{2, n b} \cdot p_{2}+P_{3, n b} \cdot p_{3}
$$

\section{2) Handoff Call Dropping Probability}

The handoff call dropping probability for $U_{1}$ connections $P_{1, h d}$ can be expressed as

$$
P_{1, h d}=\sum_{S \in \psi_{1}} \pi(S)
$$

where $\psi_{1}=\left\{S \mid R(S)+r_{1}>C\right\}$.

The handoff call dropping probability for $U_{2}$ connections $P_{2, h d}$ can be expressed as

$$
P_{2, h d}=\sum_{S \in \psi_{2}} \pi(S)
$$

where $\psi_{2}=\left\{S \mid R(S)+r_{2}>G_{1}\right\}$.

The handoff call dropping probability for $U_{3}$ connections $P_{3, h d}$ can be expressed as

$$
P_{3, h d}=\sum_{S \in \psi_{3}} \pi(S)
$$

where $\psi_{3}=\left\{S \mid R(S)+r_{3}>G_{2}\right\}$.

The overall handoff call dropping probability can be expressed as

$$
P_{h d}=P_{1, h d} \cdot q_{1}+P_{2, h d} \cdot q_{2}+P_{3, h d} \cdot q_{3}
$$

where $q_{i}=\frac{\lambda_{i, h}}{\lambda_{1, h}+\lambda_{2, h}+\lambda_{3, h}}, \quad i=1,2,3$.

\section{Parameter Optimization}

With the derived performance measures, we propose an optimization framework to balance the tradeoff among three types of services (i.e. guaranteed service, IM service and data service), and obtain the optimized system parameters. In this optimization problem, we minimize the new call blocking probability and handoff call dropping probability of IM service subjected to hard constraints respectively on the both probabilities of guaranteed service and data service. Mathematically, the system parameters are set according to the following optimization problem

$$
\begin{array}{ll}
\min _{G_{1}, G_{2}} & P_{2, n b}+\omega P_{2, h d} \\
\text { s.t. } & P_{1, n b}+\omega P_{1, h d} \leq \varepsilon_{1} \\
& P_{3, n b}+\omega P_{3, h d} \leq \varepsilon_{2}
\end{array}
$$

where $\varepsilon_{1}$ and $\varepsilon_{2}$ respectively present the constraints that the outage probability which consists of new call blocking probability and handoff call dropping probability of guaranteed services and data services. $\omega$ presents the 
weight coefficient of handoff call dropping probability. Due to the simple structure of the optimization problem (11), a search algorithm can be used to solve it.

\section{PERFORMANCE EVALUATION}

In this section, we evaluate the performance of the proposed scheme. To verify our analysis, the illustrative simulation results are shown and compared with the numerical results. The simulation is an event-drive program written in MATLAB. By considering downlink spectrum efficiency of $3.7 \mathrm{bits} / \mathrm{Hz} / \mathrm{cell}$ and subchannel bandwidth of $15 \mathrm{kHz}$, the average data rate per subchannel is set to be $55.5 \mathrm{kbps}[12]$.

We suppose that the total subchannel number in an OFDMA wireless network is 20. Each connection of guaranteed service $\left(U_{1}\right)$ occupies 10 subchannels, each connection of IM service $\left(U_{2}\right)$ occupies 2 subchannels and each connection of data service $\left(U_{3}\right)$ occupies 1 subchannel. Meanwhile, three classes of connections are generated with probability $p_{1}=0.1, p_{3}=0.3$ and $p_{3}=0.6$, respectively. And their mean call durations are set as $1 / \mu_{1}=300$, $1 / \mu_{2}=200,1 / \mu_{3}=100$. The cell radius can be set as $R=600 \mathrm{~m}$. The cell residual time $1 / \gamma=\alpha R, \alpha$ is a mobility dependent parameter which must be determined for each mobility setting, we set $\alpha=1 / 3$ in this simulation. The mean call arrival rate is $\lambda_{n}$, thus $\lambda_{1, n}=0.1 \lambda_{n}$, $\lambda_{2, n}=0.3 \lambda_{n}$ and $\lambda_{3, n}=0.6 \lambda_{n}$. The handoff call arrival rate of each service is $\lambda_{1, h}=1.5 \lambda_{1, n}, \lambda_{2, h}=\lambda_{2, n}$ and $\lambda_{3, h}=0.5 \lambda_{3, n}$.

Fig.1 (Fig.2) shows the relationship between traffic load which characterized by traffic density per user $\lambda_{n}$ and the new call blocking probabilities (handoff call dropping probabilities) of guaranteed service, IM service and data service. We let the total subchannel number $C=20$, and guard channel number $G_{1}=16, G_{2}=14$. Both the simulation and analytical results are depicted in the Fig.1 (Fig. 2). The lines represent the analytical values of new call blocking probabilities (handoff call dropping probabilities) and the marks are the simulation values of three classes of services. It is observed that the analytical results match well with the simulation results. With the value of $\lambda_{n}$ increases, probabilities of these services are increasing. By comparing both figures, we can observe that the new call blocking probability of each type of service is higher than their handoff call dropping probabilities respectively. Meanwhile, the guaranteed service which has the highest priority has the best performance. Thus, it comes up to the requirements of three types of services.

Fig.3 shows the overall new call blocking probability and the overall handoff call dropping probability in one cell. Lines represent analytical values and marks represent simulation values. From the results, we can find that they are matched very well. Further, the overall handoff call dropping probability maintains a much smaller order of magnitude as compared with the overall new call blocking probability. Thus, it means that more users with handoff calls can be admitted into a cell.

In Fig. 4 we show the searching result of optimal param-

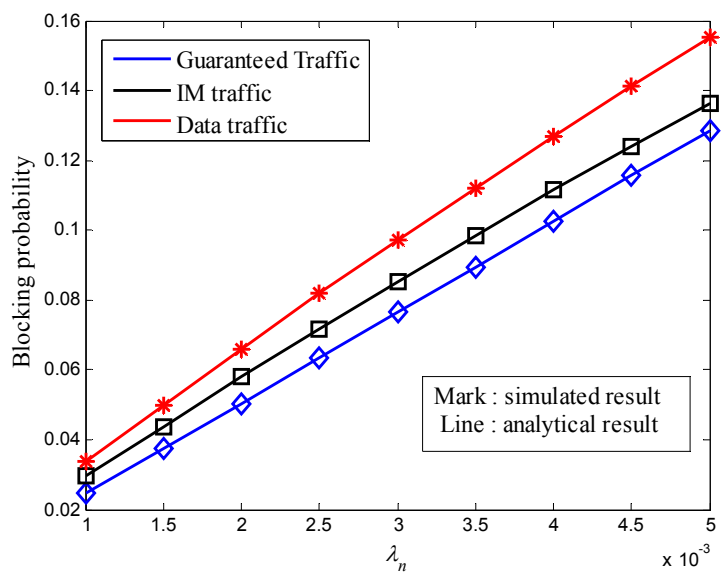

Figure 1. New call blocking probabilities of guaranteed service, IM service and data service

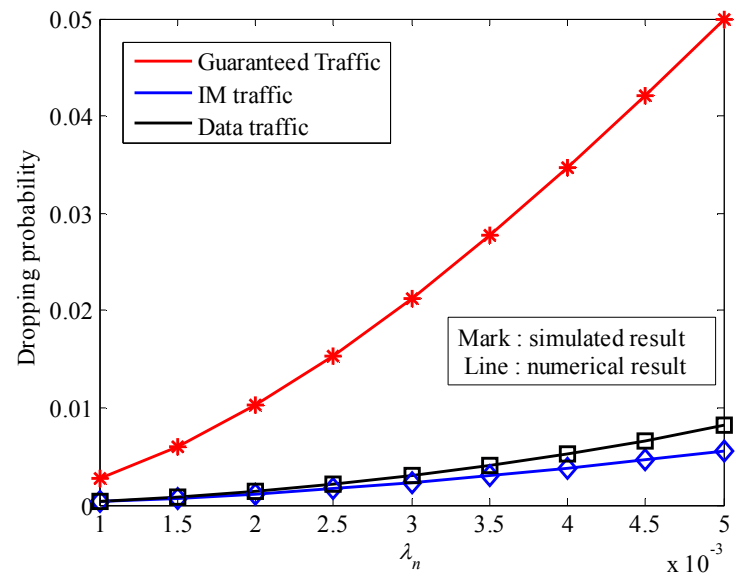

Figure 2. Handoff call dropping probabilities of guaranteed service, IM service and data service

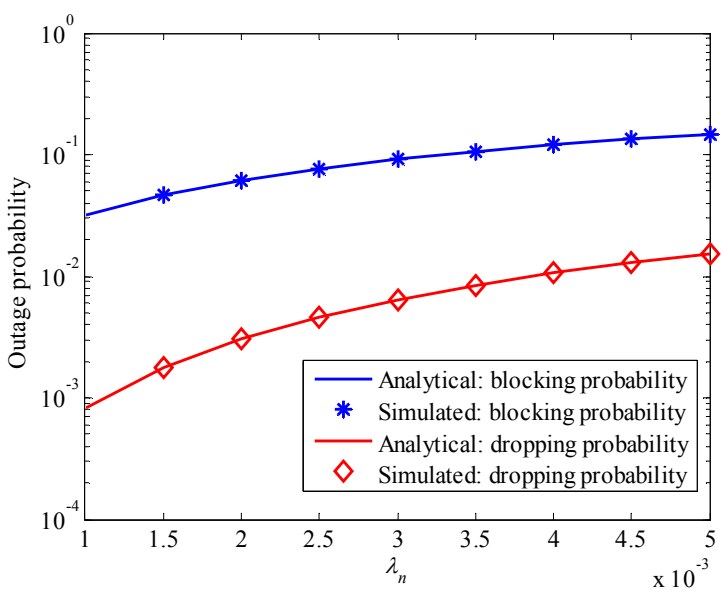

Figure 3. Outage probabilities for the overall new call blocking and the overall handoff call dropping 
eter setting for the double-threshold guard channel policy. Here, $\lambda_{n}$ is fixed to $2 \times 10^{-3}$ connection/second and $\omega$ is set to 2 . The thresholds for guaranteed traffic and data traffic are $10 \%$. Within the valid change interval (i.e. $0<G_{2}<G_{1}<C$ ), feasible solution satisfying the hard constraints are marked with star in the figure. It can be observed that there is an optimal parameter setting $\left(G_{1}=14\right.$ and $G_{2}=12$ ) and the minimal outage probability of IM traffic is $P=0.0689$. So, we can obtain the best call-level performance of IM traffic with satisfied performance of guaranteed service and data service.

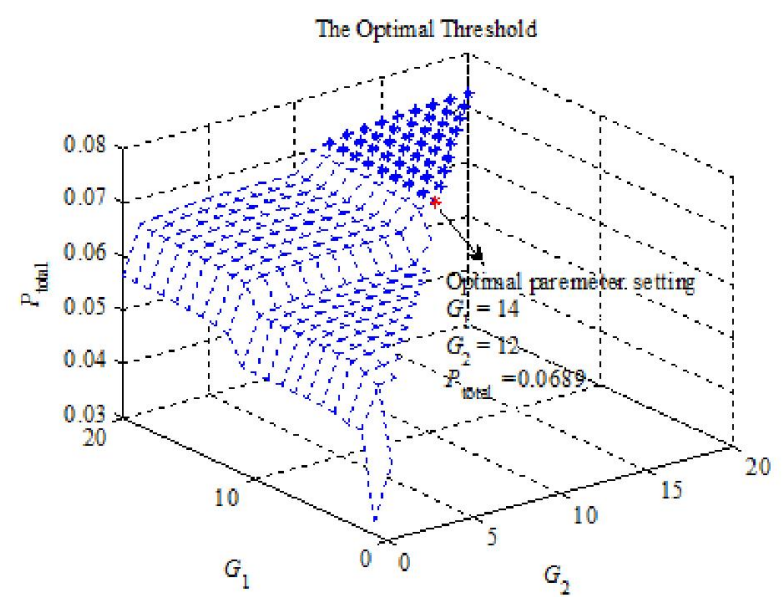

Figure 4. Search the optimal parameter setting for double-threshold guard channel policy

\section{CONCLUSION}

This paper presents an appropriate CAC scheme which admits IM traffic with conventional traffic for the next-generation OFDMA wireless network. In our scheme, we adopt the double-threshold guard channel policy for guaranteed service, IM service and data service and establish a detailed analytical model to explore the impact of the system performance. Due to the analytical values of new call blocking probability and handoff call dropping probability match well with the simulation values, this proposed scheme can be used to satisfy the call-level performance of OFDMA system with IM service. And, through adjusting the guard channel number of guaranteed service and data service dynamically, we can obtain the best QoS parameters of IM service.

\section{REFERENCES}

[1] Zhen Xiao, Guo. L, Tracey. J, "Understanding Instant Messaging Traffic Characteristics", International Conference on Distributed Computing System, pp.51, June 2007.

[2] Tung. H. Y, Tsang. K. F, Lee . L. T, Ko. K. t, "QoS for Mobile WiMAX Networks: Call Admission Control and Bandwidth Allocation", IEEE Consumer Communications and Networking Conference (CCNC), pp. 576-580, Jan. 2008.

[3] Geng-Sheng Kuo, Yao, Hui-Juan, "A QoS-Adaptive Admission Control for IEEE 802.16e-based Mobile BWA Networks", IEEE Consumer Communications and Networking Conference (CCNC), pp. 833-837, Jan. 2007.

[4] Xin Guo, Wenchao Ma, Zihua Guo, Zifeng Hou, "Dynamic Bandwidth Reservation Admission Control Scheme for the IEEE 802.16e Broadband Wireless Access Systems", IEEE Wireless Communications and Networking Conference (WCNC), pp. 3418-3423, March 2007.

[5] Kalikivayi. S, Misra. I. S, Saha. K, "Bandwidth and Delay Guaranteed Call Admission Control Scheme for QoS Provisioning in IEEE 802.16e Mobile WiMAX ", IEEE Global Telecommunications Conference (GLOBECOM), pp. 1-6, 2008.

[6] Yanfeng Guan, Aiqun Hu, "Priority-based elastic admission control algorithm of VoIP in IEEE 802.16 system", Journal on Communications, pp.23-31, 2008.

[7] Khabazian. M, Kubbar. O, Hassanein. H, "Call Admission Control with Resource Reservation for Multi-service OFDM Networks", International Conference on Computing, Networking and Communications (ICNC), pp. 781-785. Feb. 2012.

[8] Duk Kyung Kim, Griffith. D, Golmie. N, "A novel ring-based performance analysis for call admission control in wireless networks", IEEE Communications Letters, vol. 14, pp. 324-326, april 2010.

[9] Ramjee. R, Nagarajan. R, Towsley. D, "On Optimal Call Admission Control in Cellular Networks", INFOCOM '96.Fifteenth Annual Joint Conference of the IEEE Computer Societies. Networking the Next Generation. Vol. 1, pp. 43-50, Mar 1996.

[10] C. Bettstetter, "Mobility modeling in wireless networks: categorization, smooth movement, border effects", ACM Mobile Computing and Communications Review, vol. 5, no. 3, pp. 55-67,July 2001.

[11] D. K. Kim, D. K. Sung, "Characterization of soft handoff in CDMA systems", IEEE Trans. Veh. Technol, vol. 48, no. 4, pp. 1195-1202, July 1999.

[12] M. Sawahashi, Y. Kishiyama, A. Morimoto, D. Nishikawa, M. Tanno, "Coordinated multipoint transmission/reception techniques for LTE-Advanced”, IEEE Wireless Communications, pp. 26-34, June 2010 . 